\title{
Staphylococcus aureus Bacteremia Complicated by Psoas Abscess and Infective Endocarditis in a Patient with Atopic Dermatitis
}

\author{
Ichiro Tsuboi, ${ }^{1}$ Tetsuya Yumoto, ${ }^{2}$ Tatsuya Toyokawa, ${ }^{1}$ Katsunori Matsueda, ${ }^{1}$ \\ Joichiro Horii, ${ }^{1}$ Hiromichi Naito, ${ }^{2}$ and Atsunori Nakao ${ }^{2}$ \\ ${ }^{1}$ Department of Gastroenterology, National Hospital Organization Fukuyama Medical Center, 4-14-17, Okinogamicho, \\ Fukuyama-shi, Hiroshima 720-8520, Japan \\ ${ }^{2}$ Advanced Emergency and Critical Care Medical Center, Okayama University Hospital, 2-5-1 Kita-ku, Shikata-cho, \\ Okayama-shi, Okayama 700-8558, Japan
}

Correspondence should be addressed to Tetsuya Yumoto; tyumoto@cc.okayama-u.ac.jp

Received 2 September 2017; Accepted 21 November 2017; Published 10 December 2017

Academic Editor: Larry M. Bush

Copyright (C) 2017 Ichiro Tsuboi et al. This is an open access article distributed under the Creative Commons Attribution License, which permits unrestricted use, distribution, and reproduction in any medium, provided the original work is properly cited.

\begin{abstract}
The close relationship between atopic dermatitis (AD) and infective endocarditis (IE) has been implicated. Staphylococcus aureus colonization is frequently seen observed in $\mathrm{AD}$ patients' skin lesions. Although a case of IE due to S. aureus bacteremia in an $\mathrm{AD}$ patient has been sporadically reported, a case of $S$. aureus bacteremia complicated by psoas abscess and IE has not been previously reported. A 42-year-old man with a history of AD presented to our hospital complaining of fever, fatigue, chills, lower right back pain, and poor appetite for a week. His blood cultures showed growth of $S$. aureus. On day 3 , the patient presented acute cardiac failure and was diagnosed with IE based on echocardiogram examination. Since the patient's cardiac failure did not respond to medication, an emergency surgery was performed on the fourth day of hospitalization. The patient underwent successful surgical treatment of the heart lesions and subsequent percutaneous drainage of psoas abscess and received intensive antibiotics, which successfully improved his condition. Our report emphasizes awareness of the association between $\mathrm{AD}$ and invasive $S$. aureus infections.
\end{abstract}

\section{Introduction}

Atopic dermatitis $(\mathrm{AD})$, an ongoing, relapsing, eczematous skin disorder marked by inflammation and pruritus, is caused by various specific allergens and unspecific stimuli. Being one of the most prevalent skin diseases, $\mathrm{AD}$ affects up to $1-3 \%$ of adults and $20 \%$ of children and in most nations all over the world [1].

Patients with $\mathrm{AD}$ are more vulnerable to infection due to impaired skin barrier. Staphylococcus aureus, in particular, is frequently seen in $\mathrm{AD}$ patients' skin lesions [2]. An association between severe $\mathrm{AD}$ and acute bacterial endocarditis after recurring staphylococcal skin infection was first reported by Pike and Warner [3]. Since then, a close relationship between $\mathrm{AD}$ and infective endocarditis (IE) has been advocated [4].

Although a substantial number of case reports have depicted the simultaneous presence of IE and $\mathrm{AD}, S$. aureus bacteremia complicated with both psoas abscess and IE has not been previously described in a patient with $\mathrm{AD}$ [5-7]. Herein, we report a case of psoas abscess and IE caused by $S$. aureus in a patient with $\mathrm{AD}$. Sharing our experience with readers may help clinicians develop a proper and early diagnostic strategy, a key to the successful management of invasive $S$. aureus infections in $\mathrm{AD}$ patients.

\section{Case Presentation}

A 42-year-old man with a history of $\mathrm{AD}$ presented to our hospital. His AD skin lesion was treated with topical corticosteroids (diflucortolone valerate). $\mathrm{He}$ denied any scratches or recent skin injuries and had never used intravenous drugs. He complained of fever, fatigue, chills, lower right back pain, and poor appetite for a week. He had no previous history of heart disease or rheumatic fever. On physical examination, his body temperature was $38.3^{\circ} \mathrm{C}$, 


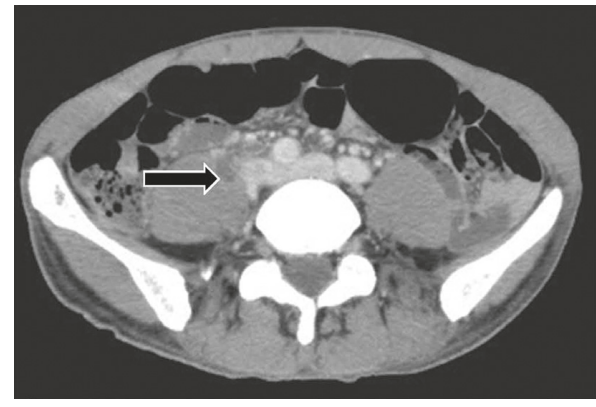

(a)

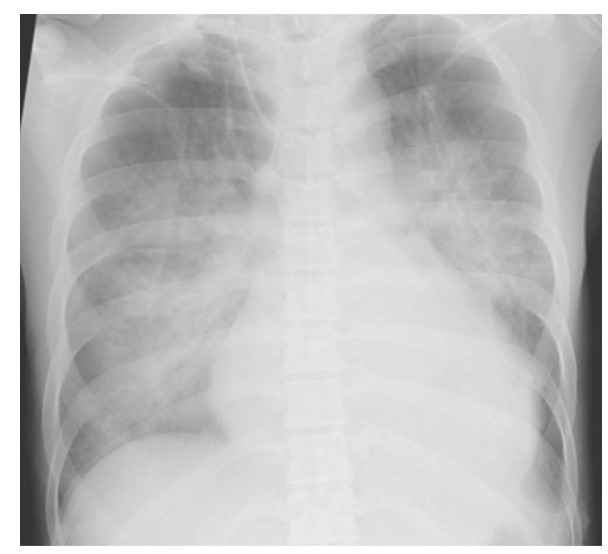

(b)

Figure 1: (a) There was a low-density foci with enhancement of the wall in the patient's right iliopsoas muscle, raising suspicion of iliopsoas abscess. (b) The result of chest radiography on day 3 showed severe cardiomegaly, measured as a cardiothoracic ratio of $61 \%$ with pulmonary edema.

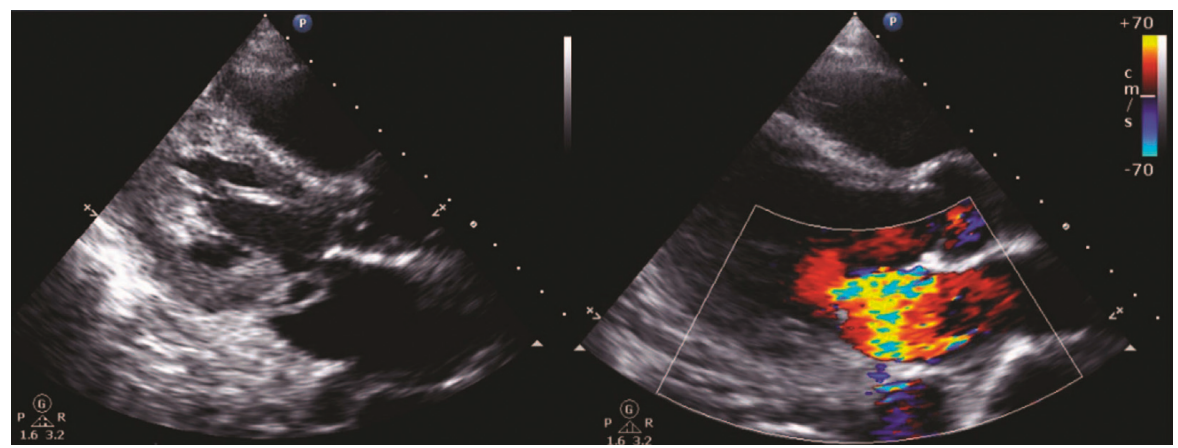

(a)

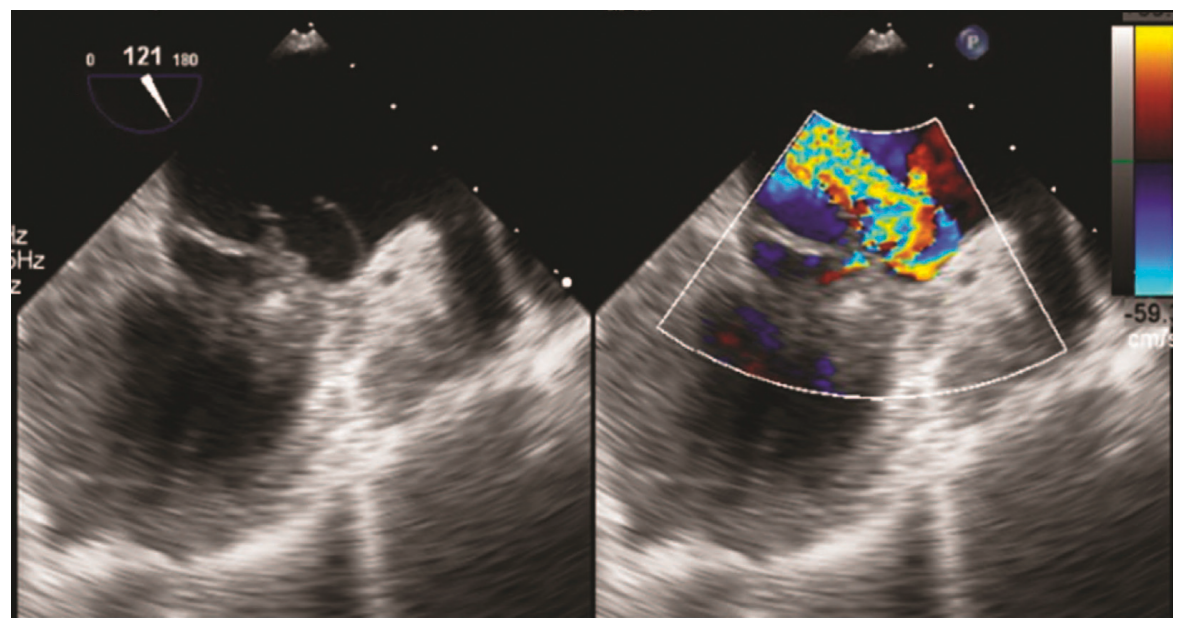

(b)

FIgUre 2: (a) In parasternal long-axis view, wall motion was normokinetic and ejection fraction was over $60 \%$. Posterior leaflet of mitral valve (P1) had a valvular aneurysm and perforation of the valvular aneurysm. Severe mitral regurgitation is shown in color Doppler echo imaging. (b) Transesophageal echocardiography showed that posterior leaflet of mitral valve (P1) had a valvular aneurysm, with perforation of the valvular aneurysm and severe mitral regurgitation, but did not have vegetation or abscess of the mitral valve annulus.

pulse rate was $84 / \mathrm{min}$, blood pressure was $106 / 76 \mathrm{mmHg}$, and respiratory rate was 18 breaths/min. His neurologic examination was unremarkable; he had a Glasgow Coma Scale score of 15 without altered sensorium, meningeal signs, or focal deficits. He had no conjunctival pallor or icterus or jugular vein distention. Cardiovascular and respiratory exam showed that his murmur and breath sounds were normal. 
A skin survey demonstrated no signs such as Osler's node or Janeway lesion. Laboratory tests indicated a white blood cell count of $3.5 \times 10^{3} / \mu \mathrm{l}$, platelet count of $98 \times 10^{3} / \mu \mathrm{l}$, and C-reactive protein level of $37.29 \mathrm{mg} / \mathrm{dl}$. His electrocardiogram and chest X-ray were unremarkable, without an enlarged heart shadow or a consolidation. Contrast-enhanced computed tomography (CT) revealed a low-density mass in the iliopsoas muscle, raising suspicion of an iliopsoas abscess (Figure 1(a)). Two sets of blood cultures were obtained. Based on the preliminary clinical diagnosis of systemic inflammatory response associated with primary psoas abscess, empirical administration of intravenous meropenem was initiated at 1 gram every eight hours.

The day following admission, the patient complained of chest discomfort and continued fever. Electrocardiogram and chest X-ray did not demonstrate any abnormal findings. The blood cultures exhibited growth of $S$. aureus sensitive to vancomycin; the antimicrobial treatment was then switched from meropenem to vancomycin at 1 gram every 12 hours. Three days after admission, his blood pressure and heart rate were $100 / 76 \mathrm{mmHg}$ and $160 / \mathrm{min}$, respectively, with blood lactate levels of $1.1 \mathrm{mmol} / \mathrm{L}$. Fluid resuscitation and inotropic support to maintain circulation were initiated. Chest X-ray revealed cardiomegaly with pulmonary edema (Figure 1(b)). Due to increasing chest discomfort, the patient was transferred to the intensive care unit and required mechanical ventilation and endotracheal intubation with positive airway pressure. Transthoracic echocardiography in the parasternal long-axis view revealed normal wall motion and fair ejection fraction $>60 \%$. The posterior leaflet of the mitral valve had a valvular aneurysm with perforation. Color Doppler echocardiography revealed severe mitral regurgitation (Figure 2(a)). Cardiomegaly was observed due to enlargement of left atrium and left ventricle, although it was uncommon in the setting of acute mitral regurgitation. Subsequent transesophageal echocardiography notably failed to show any obvious vegetation or abscess of the mitral valve annulus (Figure 2(b)). As these results met both the modified Duke Criteria and the Jones criteria, we made the diagnosis of IE. Since conservative medical management, including antibiotic administration, was suboptimal for curative treatment, we concluded that valvular surgery was required. The patient was transferred to the cardiovascular surgery department and underwent emergency surgery on the third day after admission. Surgery revealed the presence of vegetations on the destroyed left cusps, and the infection had reached the annulus. The anterior commissure leaflet was resected together with the vegetation and ruptured chordae tendineae, followed by mitral valve replacement.

CT-guided percutaneous drainage of the abscess in the right psoas muscle was successfully carried out two weeks after the surgery. The antibiotic treatment with vancomycin in combination with ceftriaxone was continued for four weeks, followed by sole antibiotic therapy with ceftriaxone, which was discontinued after three consecutive blood cultures had been confirmed negative. The postoperative course was unremarkable, and the patient was discharged from the institution 45 days later.

\section{Discussion}

This is the first report of $S$. aureus bacteremia complicated with both psoas abscess and IE in a patient with AD. Our report highlights the fact that $\mathrm{AD}$ can predispose to development of invasive $S$. aureus infections including psoas abscess and IE.

$\mathrm{AD}$ is the most common persistent inflammatory skin disorder characterized by intense pruritus and recurrent erythema. Patients with AD exhibit significant skin barrier disruption which leads to increased susceptibility to infection [8]. The human skin innate immune system includes the antimicrobial peptides $\beta$-defensins (HBD-2) and cathelicidins (LL-37), which are induced by inflammation and have antimicrobial, antifungal, and antiviral properties. These peptides are deficient in $\mathrm{AD}$ patients, resulting in increased susceptibility to microorganisms and providing an inviting environment for invasion, proliferation, and colonization of $S$. aureus $[9,10]$.

As skin colonization with $S$. aureus is universal in $\mathrm{AD}$ patients, invasive $S$. aureus infections including IE occasionally occur in patients with $\mathrm{AD}$ [11]. As for IE, S. aureus is the most common microorganism responsible for IE. Risk factors of IE due to $S$. aureus include prosthetic heart valve, injecting drug use, and persistent bacteremia [12]. Although there is no clear evidence of a higher frequency of the coexistence of IE and AD, IE should be known as an important complication in this familiar skin disorder [4]. Higher C-reactive protein and persistent fever in the setting of $S$. aureus bacteremia are associated with IE as the present case showed $[12,13]$.

IE due to $S$. aureus more frequently causes complications including septic embolization and metastatic infection than other pathogens [14]. Horino et al. described that metastatic infections such as psoas abscess, spondylitis, IE, pulmonary abscess, and epidural abscess occurred in 14 of 73 patients with $S$. aureus bacteremia [13]. From the aspect of psoas abscess, since it can present with IE, patients with psoas abscess should be investigated for IE particularly caused by S. aureus [15]. To our knowledge, this is the first case of psoas abscess and IE due to $S$. aureus bacteremia in a patient with $\mathrm{AD}$ and otherwise healthy. Invasive $S$. aureus infections including IE and metastatic abscess should be considered in $\mathrm{AD}$ patients presenting with prolonged fever or fatigue.

\section{Conclusion}

$\mathrm{AD}$ can be a potential risk factor for invasive $S$. aureus infections including IE and psoas abscess. Physicians should be aware of the association between $\mathrm{AD}$ and invasive $S$. aureus infections to develop a proper and early diagnostic strategy.

\section{Conflicts of Interest}

The authors have no conflicts of interest or financial disclosure to announce.

\section{References}

[1] J. Ring, A. Alomar, T. Bieber et al., "Guidelines for treatment of atopic eczema (atopic dermatitis) Part I," Journal of the 
European Academy of Dermatology and Venereology, vol. 26, no. 8, pp. 1045-1060, 2012.

[2] C. Hauser, B. Wuethrich, L. Matter, J. A. Wilhelm, W. Sonnabend, and K. Schopfer, "Staphylococcus aureus skin colonization in atopic dermatitis patients," Dermatologica, vol. 170, no. 1, pp. 35-39, 1985.

[3] M. G. Pike and J. O. Warner, "Atopic dermatitis complicated by acute bacterial endocarditis," Acta Paediatrica Scandinavica, vol. 78, no. 3, pp. 463-464, 1989.

[4] N. Kondo and I. Fukuda, "Rising awareness of the relationship between infective endocarditis and atopic dermatitis," Circulation Journal, vol. 77, no. 7, pp. 1693-1694, 2013.

[5] S. A. Grabczynska and R. Cerio, "Infective endocarditis associated with atopic eczema," British Journal of Dermatology, vol. 140, no. 6, pp. 1193-1194, 1999.

[6] K. Onoda, H. Mizutan, T. Komada et al., "Atopic dermatitis as a risk factor for acute native valve endocarditis," Journal of Heart Valve Disease, vol. 9, no. 3, pp. 469-471, 2000.

[7] G. Mohiyiddeen, I. Brett, and E. Jude, "Infective endocarditis caused by Staphylococcus aureus in a patient with atopic dermatitis: a case report," Journal of Medical Case Reports, vol. 2, no. 1, p. 143, 2008.

[8] C. L. Jinnestal, E. Belfrage, O. Back, A. Schmidtchen, and A. Sonesson, "Skin barrier impairment correlates with cutaneous Staphylococcus aureus colonization and sensitization to skin-associated microbial antigens in adult patients with atopic dermatitis," International Journal of Dermatology, vol. 53, no. 1, pp. 27-33, 2014.

[9] P. Y. Ong, T. Ohtake, C. Brandt et al., "Endogenous antimicrobial peptides and skin infections in atopic dermatitis," New England Journal of Medicine, vol. 347, no. 15, pp. 1151$1160,2002$.

[10] I. Wanke, Y. Skabytska, B. Kraft, A. Peschel, T. Biedermann, and B. Schittek, "Staphylococcus aureus skin colonization is promoted by barrier disruption and leads to local inflammation," Experimental Dermatology, vol. 22, no. 2, pp. 153-155, 2013.

[11] S. Benenson, O. Zimhony, D. Dahan et al., "Atopic dermatitis-a risk factor for invasive Staphylococcus aureus infections: two cases and review," The American Journal of Medicine, vol. 118, no. 9, pp. 1048-1051, 2005.

[12] V. Le Moing, F. Alla, T. Doco-Lecompte et al., "Staphylococcus aureus bloodstream infection and endocarditis-a prospective cohort study," PLoS One, vol. 10, no. 5, article e0127385, 2015.

[13] T. Horino, F. Sato, Y. Hosaka et al., "Predictive factors for metastatic infection in patients with bacteremia caused by methicillin-sensitive Staphylococcus aureus," The American Journal of the Medical Sciences, vol. 349, no. 1, pp. 24-28, 2015.

[14] V. G. Fowler Jr., J. M. Miro, B. Hoen et al., "Staphylococcus aureus endocarditis: a consequence of medical progress," JAMA, vol. 293, no. 24, p. 3012, 2005.

[15] T. J. Cahill and B. D. Prendergast, "Infective endocarditis," The Lancet, vol. 387, no. 10021, pp. 882-893, 2016. 


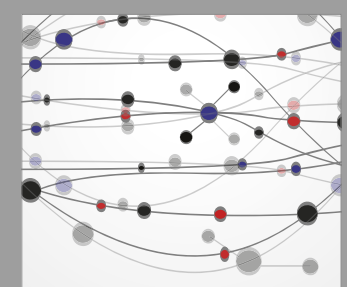

The Scientific World Journal
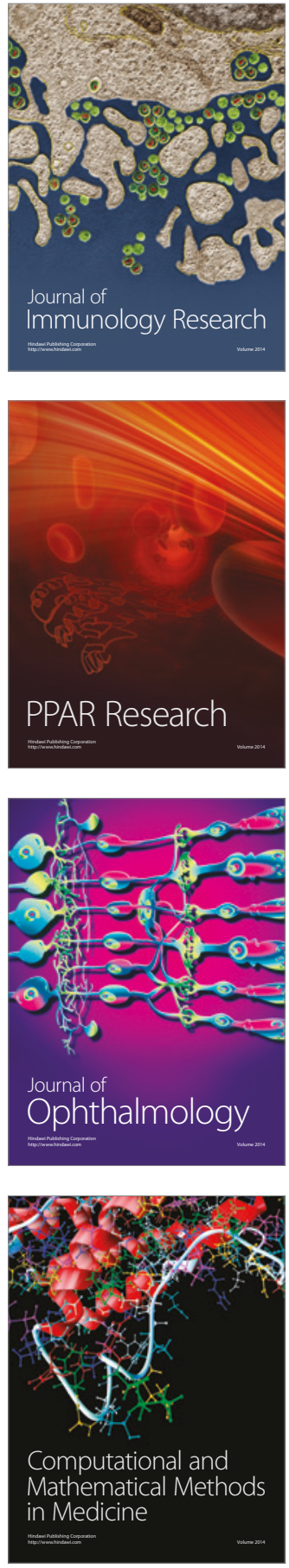

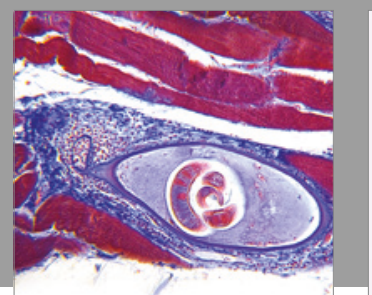

Gastroenterology Research and Practice
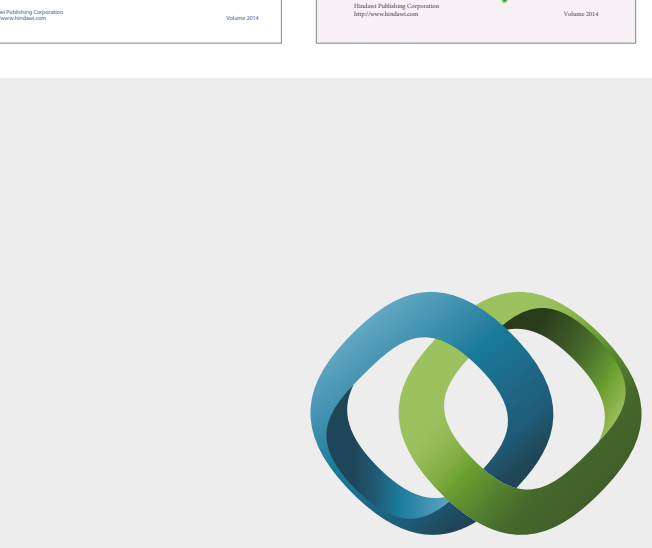

\section{Hindawi}

Submit your manuscripts at

https://www.hindawi.com
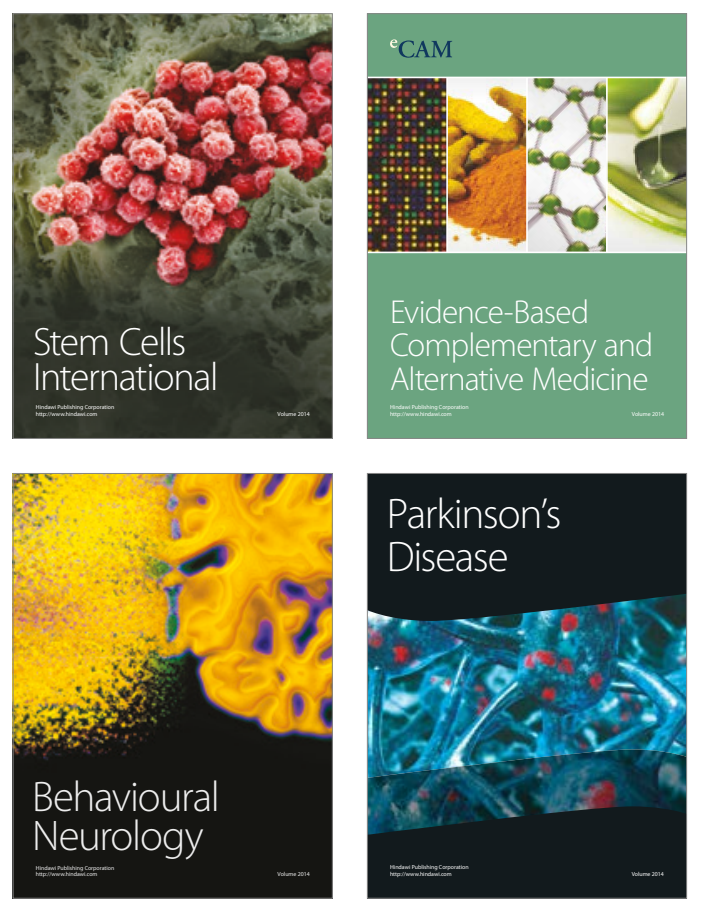
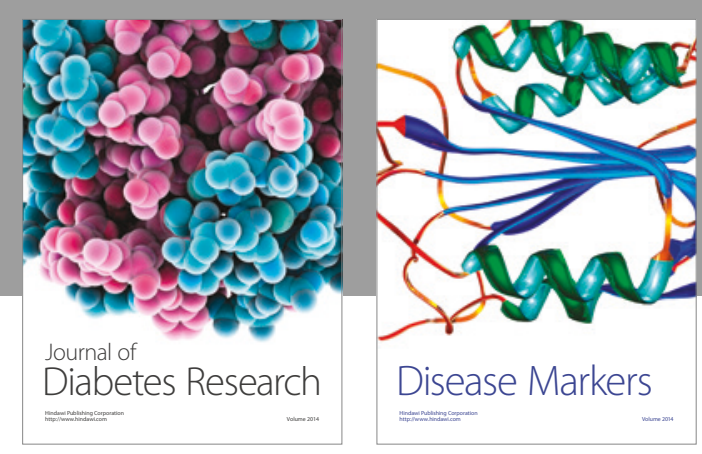

Disease Markers
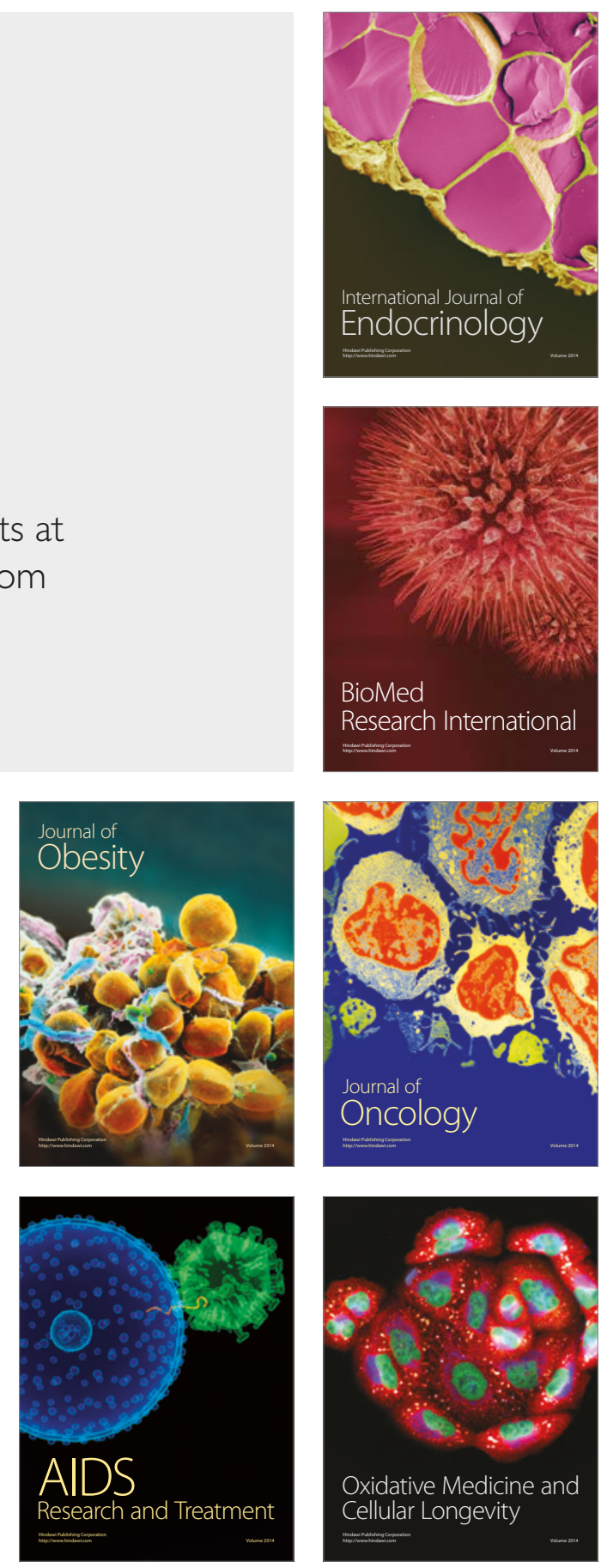